\title{
SOP Fissuren- und Grübchenversiegelung
}

Jan Kühnisch, Roswitha Heinrich-Weltzien, Reinhard Hickel

\author{
Karies gehört nach wie vor zu den häufigen Erkrankungen in der Bevölkerung. \\ Dabei konzentriert sich der Kariesbefall im Kindes- und Jugendalter auf die \\ bleibenden Molaren und deren Fissuren und Grübchen. Um einer Kariesinitiation \\ wirksam vorzubeugen oder vorhandene Initialstadien zu arretieren, steht die \\ Fissuren- und Grübchenversiegelung zur Verfügung.
}

\section{Einleitung}

Unter einer Versiegelung wird der präventive Verschluss der kariesanfälligen Fissuren und Grübchen an Molaren verstanden, um einer Kariesinitiation vorzubeugen und/ oder kariöse Frühstadien zu arretieren [1]. Die Fissurenund Grübchenversiegelung ist damit eine zahnflächenspezifische Präventionsmaßnahme. Ziel der Maßnahmen ist die Umgestaltung des plaqueretentiven Fissurenreliefs in eine prophylaxefähige Oberfläche. Darüber hinaus ergänzen eine zahngesunde Ernährung, adäquate häusliche Mundhygienemaßnahmen sowie indikationsgerechte häusliche und professionelle Fluoridapplikationen [2] als wirksame und evidenzbasierte Maßnahmen das Portfolio der Präventivbetreuung.

Die Versiegelung kann prinzipiell an allen Zähnen mit Fissuren oder Grübchen in der primären und bleibenden Dentition angewendet werden. Da der größte präventive Nutzen im Vergleich zu anderen Zahngruppen, wie Milchzähnen, bleibenden Front- und Eckzähnen sowie Prämolaren, an den bleibenden Molaren zu erwarten ist, ist die Abrechenbarkeit der Versiegelung (IP5-Position des BEMA) in der Bundesrepublik Deutschland auf diese Zähne begrenzt und damit priorisiert.

Die Thematik war und ist Gegenstand vielfältigster Metaanalysen [3-5], klinischer Empfehlungen [1,6-9], klinischer Studien und Laboruntersuchungen. Die verfügbare Literatur wird darüber hinaus in der S3-Leitlinie „Fissuren- und Grübchenversiegelung“ zusammengefasst [10]. Ziel des vorliegenden Beitrages ist es, den im Rahmen der S3-Leitlinie dokumentierten klinischen Workflow im Sinne eines standardisierten Arbeitsablaufes (SOP - Standard Operating Procedure) darzustellen.

\section{Indikation und Kontraindikationen}

Die Indikationsstellung zur Fissuren- und Grübchenversiegelung erfolgt auf Grundlage der visuellen und ergänzenden Kariesdiagnostik sowie einer Kariesrisiko-Beurteilung ( $>$ Abb. 1). In Abhängigkeit von der zahnflächenbezogenen Diagnose - gesund, nicht kavitierte kariöse Läsion oder (Mikro)Kavitation/Dentinkaries - wird die Indikation zur präventiven Fissuren- und Grübchenversiegelung bzw. minimal invasiven Füllungstherapie gestellt. An gesunden Fissuren und Grübchen sowie nicht kavitierten kariösen Läsionen ist die Fissuren- und Grübchenversiegelung prinzipiell indiziert. Im Fall von Kavitationen wird die Füllungstherapie als Vorgehen der Wahl angesehen und diese defektorientiert vorgenommen.

Das allgemeine Kariesrisiko gilt als eine weitere Einflussvariable bei der Entscheidungsfindung, welche insbesondere an (noch) gesunden Fissuren und Grübchen zum Tragen kommt. Bei kariesfreien Patienten kann aus heutiger Sicht auf die Fissuren- und Grübchenversiegelung oft verzichtet werden, da die Wahrscheinlichkeit einer okklusalen Kariesentwicklung bei sichergestellter präventiver Betreuung, z. B. durch eine zahngesunde Ernährungsweise, (supervidiertes) tägliches Zähneputzen mit Fluoridzahnpasta und indikationsgerechten Fluoridlackapplikationen im professionellen Umfeld, sowie regelmäßigen Kontrolluntersuchungen als gering eingeschätzt wird. Nichtsdestotrotz wird an Zähnen mit einem erhöhten zahnflächenspezifischen Risiko, z. B. bei tiefen Fissuren, die Versiegelung auch bei Nicht-Kariesrisiko-Patienten empfohlen. Für Kinder, Jugendliche und junge Erwachsene mit einem erhöhten Kariesrisiko ist die Fissuren- und Grübchenversiegelung auch an gesunden Molaren Bestandteil der kariespräventiven Betreuungsstrategie. Bei Erwachsenen und älteren Patienten kann die Indikation zur Versiegelung restriktiver gestellt werden. 


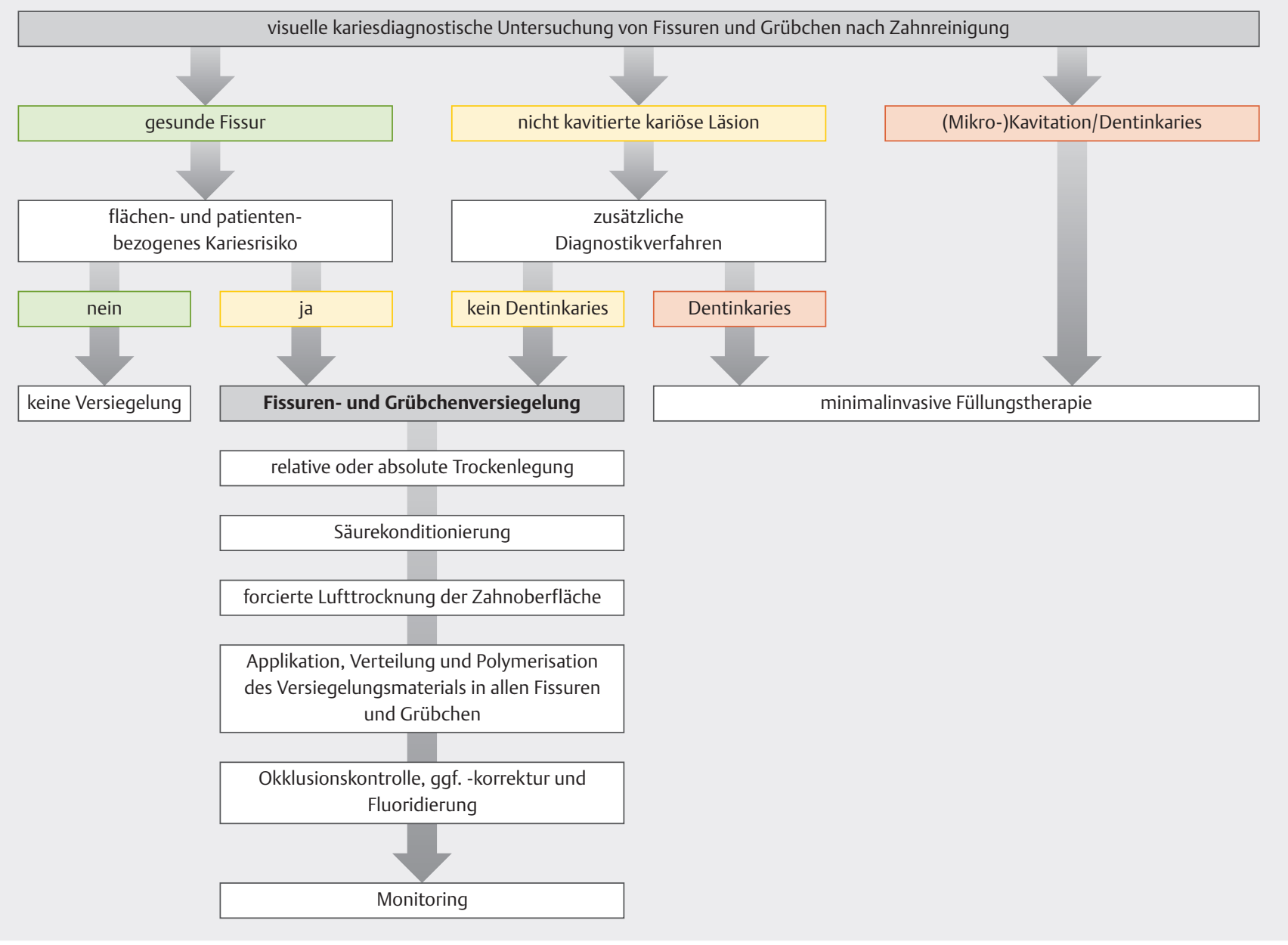

- Abb. 1 Darstellung der Entscheidungsfindung und klinischen Durchführung der Fissuren- und Grübchenversiegelung.

Zusammenfassend kann formuliert werden, dass die Indikation zur Fissuren- und Grübchenversiegelung an bleibenden Molaren in folgenden klinischen Situationen zu stellen ist:

- Kariesfreie Fissuren und Grübchen bei Patienten mit einem erhöhtem Kariesrisiko. Dazu zählen z. B. Patienten mit Karieserfahrung im Milch- und bleibendem Gebiss.

- Kariesfreie Fissuren und Grübchen mit einem anatomisch kariesanfälligen Fissurenrelief.

- Fissuren und Grübchen mit nicht kavitierten kariösen Läsionen unabhängig von der Kariesrisiko-Einschätzung.

- Fissuren und Grübchen bei Patienten mit Allgemeinerkrankungen bzw. körperlichen und/oder geistigen Behinderungen, die eine effektive tägliche Mundhygiene nur begrenzt umsetzen können und damit einem erhöhten Kariesrisiko unterliegen.

- Partiell oder vollständig verloren gegangene Fissurenversiegelungen sollten bei unverändertem Kariesrisiko repariert bzw. erneuert werden.
- Relative Kontraindikationen zur Fissuren- und Grübchenversiegelung bestehen in folgenden Situationen:

- Ist der betreffende Zahn noch nicht vollständig in die Mundhöhle durchgebrochen und sind die Okklusalfläche bzw. die palatinalen/bukkalen Grübchen nicht oder nur begrenzt einer adäquaten Trockenlegung bzw. Instrumentierung zugänglich, sollte auf die Versiegelung vorerst verzichtet werden.

- Bei Zähnen mit einer nachgewiesenen Dentinkaries im Bereich der Fissuren bzw. Grübchen ist die Versiegelung kontraindiziert und eine minimal invasive Füllungstherapie angezeigt.

Eine absolute Kontraindikation zur Fissuren- und Grübchenversiegelung besteht bei einer nachgewiesenen Allergie gegenüber Versiegelungsmaterialien oder einzelnen Materialbestandteilen. 


\section{Klinisches Vorgehen}

Die Fissuren- und Grübchenversiegelung ist eine einfache, nicht invasive und in wenigen Minuten durchzuführende Behandlungstechnik. Nichtsdestotrotz sind einige Behandlungsschritte erforderlich, welche durch qualifiziertes Personal unter vierhändigem Arbeiten also unter der Mitwirkung einer Assistenz - erbracht werden müssen.

In einem ersten Arbeitsschritt ist eine saubere Zahnoberfläche herzustellen, da diese einerseits Voraussetzung für die diagnostische Untersuchung ist und andererseits optimale Bedingungen für die Konditionierung der Zahnoberfläche garantiert. Dabei ist die Zahnreinigung mit einem rotierenden Bürstchen mit bzw. ohne Verwendung einer Prophylaxepaste heute als das Routinevorgehen anzusehen. Dieses ist sowohl in einer Vielzahl klinischer Untersuchungen erprobt und unter Praxisbedingungen einfach, schnell und kindgerecht durchführbar.

Als eine weitere Voraussetzung für den Behandlungserfolg ist eine sichere Trockenlegung des Arbeitsfeldes während der gesamten Versiegelungsprozedur zu nennen. Kann kein vierhändiges Arbeiten mit relativer Trockenlegung und effektiver Absaugung im Praxisalltag umgesetzt werden, wird die Applikation der Fissurenund Grübchenversiegelung unter Zuhilfenahme von Kofferdam empfohlen. Aus praktischer Sicht ist festzustellen, dass die absolute Trockenlegung zwar ein sicheres Arbeiten ermöglicht, aber nicht immer von Kindern und Jugendlichen akzeptiert wird. Der Einfluss der Trockenlegung auf die Retentionsrate von Fissuren- und Grübchenversiegelungen wurde in einzelnen vergleichenden klinischen Untersuchungen verifiziert. Die dokumentierten Retentionsraten deuten dabei auf eine Gleichwertigkeit der absoluten und relativen Trockenlegung hin. Daher kann argumentiert werden, dass im klinischen Alltag das weniger aufwendige und kindgerechte Vorgehen zu favorisieren ist.

Der erste klinische Arbeitsschritt im Anschluss an die Reinigung und Trockenlegung des zu versiegelnden Zahnes ist die Konditionierung der Schmelzoberfläche mit dem Ziel, die äußere aprismatische Schmelzschicht zu modifizieren. Dies ist erforderlich, um einen adhäsiven Verbund zwischen dem Zahnschmelz und dem methacrylatbasierten Versiegelungsmaterial herzustellen. Obwohl dies prinzipiell mit verschiedenen Methoden möglich ist, hat sich im klinischen Alltag die Konditionierung mit 35\%iger Ortho-Phosphorsäure in Gelform mehrheitlich durchgesetzt. Gele zeichnen sich hier durch eine kontrollierbare und ortsständige Applikation aus. Das resultierende Ätzmuster ist Voraussetzung für die mikromechanische Verankerung des Versiegelungsmaterials und führt zur Freilegung der darunter liegenden Schmelzprismen, mit welchen sich der hydrophobe Versiegelungs- kunststoff verzahnt. Gegenstand andauernder Diskussionen ist die Einwirkzeit der Säure am Zahnschmelz. Obwohl eine Verkürzung der Säurekonditionierung von Beginn der Verfügbarkeit der Fissuren- und Grübchenversiegelung immer wieder diskutiert wurde, bleibt zu konstatieren, dass dieser Ansatz einerseits nur durch wenige klinische Daten gestützt wird, die andererseits ein heterogenes Bild in Bezug auf die Retention und damit auf das Überleben der Versiegelung dokumentieren. Daher wird empfohlen, die Säure am unbehandelten Zahnschmelz des bleibenden Zahnes für 30-60 Sekunden einwirken zu lassen. Eine Verkürzung der Säurekonditionierung auf unter 30 Sekunden kann gegenwärtig nicht empfohlen werden. Nach gründlichem Absprayen der Säure und forcierter Trocknung muss eine kreidig weiße Schmelzoberfläche sichtbar sein. Dieses Merkmal gilt als Kontrolle für einen erfolgreichen Ätzvorgang vor der Applikation des Versiegelungsmaterials.

Lichtpolymerisierende Versiegeler werden als Einkomponentenmaterialien heute klinisch bevorzugt zum Einsatz gebracht, da diese in der Anwendung wenig techniksensitiv sind und die sofortige Lichtpolymerisation eine kurze Behandlungszeit ermöglicht. Aus praktischer Sicht ist zu ergänzen, dass eine sparsame Applikation ohne Materialüberschuss des in der Regel dünnfließenden Versiegelungsmaterials in allen (Para)Fissuren und Grübchen gefordert wird. Überschüssiges Material kann leicht mit einem Brush-Stick vor der abschließenden Lichtpolymerisation entfernt werden. Zur Lichtpolymerisation können handelsübliche Halogen- oder LED-Lampen mit ausreichender Intensität genutzt werden. Beide Lampentypen sind als gleichwertig anzusehen. Die produktabhängige Polymerisationszeit - typischerweise 20 Sekunden - muss eingehalten werden. Bei einem kleinen Durchmesser des Lichtaustrittsfensters müssen mehr als einmal und räumlich versetzt die versiegelten Flächen belichtet werden. Im Anschluss an die Polymerisation soll eine Okklusionskontrolle und im Fall von okklusalen Vorkontakten eine Korrektur erfolgen. Grundsätzlich wird die Entfernung der oberflächlich nicht polymerisierten Kunststoffschicht mit einer kurzen Politur empfohlen. Die Remineralisation geätzter, aber nicht versiegelter Schmelzareale wird durch die Lokalapplikation eines Fluoridpräparates gefördert.

Aufgrund eines möglichen Retentionsverlustes wird eine regelmäßige Kontrolle vorhandener Fissuren- und Grübchenversiegelungen alle 6-12 Monate empfohlen. Die Kontrollintervalle sollten bei Patienten mit einem hohen Kariesrisiko 12 Monate nicht überschreiten. Im Fall eines vollständigen oder teilweisen Retentionsverlustes erfolgt die Indikationsstellung und Applikation der Fissuren- und Grübchenversiegelung analog der oben formulierten Vorgehensweise. Das verbliebene Versiegelungsmaterial ist hinsichtlich seiner Retention zu prüfen. Eine vollständige Entfernung fest anhaftender Materialreste ist nicht erforderlich. 


\section{ZUSAMMENFASSUNG}

Zur Kariesvorbeugung steht die Fissuren- und Grübchenversiegelung als effektive, zahnflächenspezifische Maßnahme zur Verfügung, welche vorrangig im Kindes- und Jugendalter zum Einsatz kommt. Der kariespräventive Nutzen wurde in systematischen Literaturübersichten [3] herausgearbeitet. Voraussetzung für den Erfolg dieses Vorgehens ist immer die vollständige Versiegelung des gesamten Fissurenreliefs unter Einhaltung der entsprechenden Arbeitsschritte und Wahrung des Qualitätsmanagements.

\section{Interessenkonflikt}

Die Autorinnen/Autoren hatten während der letzten 3 Jahre wirtschaftliche oder persönliche Verbindungen im genannten Sinne.

\section{Autorinnen/Autoren}

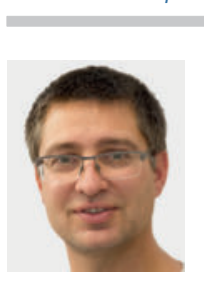

\section{Jan Kühnisch}

Prof. Dr. med. dent. 1991-1996 Studium der Zahnmedizin an der Universität Leipzig und FSU Jena, 1998-1999 Assistenzzeit, 2000 Wissenschaftlicher Assistent an der FSU Jena, 2003 Spezialisierung im Fachbereich „Kinder- und Jugendzahnheilkunde“, seit 2004 Mitarbeiter an der Poliklinik für Zahnerhaltung und Parodontologie der LMU München, 2006 Ernennung zum Oberarzt, 2008/09 Habilitation und Ernennung zum Privatdozenten, 2015 Ernennung zum APL-Professor.

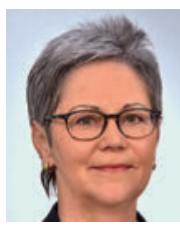

\section{Roswitha Heinrich-Weltzien}

Prof. Dr. med. dent. 1969-1974 Studium der Zahnmedizin an der FSU Jena, 1979 Promotion und Fachzahnärztin Kinderzahnheilkunde, 1987 Habilitation, Oberärztin und Privatdozentin, von 1974-1998 Mitarbeiterin der Poliklinik für Präventive Zahnheilkunde und Kinderzahnheilkunde an der Medizinischen Akademie Erfurt, 2000 Ernennung zur APL-Professorin, von 2009-2018 Komm. Direktorin der Poliklinik für Präventive Zahnheilkunde und Kinderzahnheilkunde am Universitätsklinikum Jena.

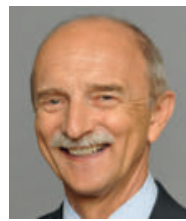

\section{Reinhard Hickel}

Prof. Dr. med. dent. Studium, Promotion (1981), Oberarzt-Ernennung (1986) und Habilitation (1988) an Klinik für Zahnerhaltung und Parodontologie der Universität ErlangenNürnberg, ab 1992 Ordinarius und Direktor der Poliklinik für Zahnerhaltung und Parodontologie und Kinderzahnheilkunde, LMU München.

\section{Korrespondenzadresse}

Prof. Dr. med. dent. Jan Kühnisch

Poliklinik für Zahnerhaltung und Parodontologie

Kinder- und Jugendbehandlung

Goethestraße 70

80336 München

Deutschland

jkuehn@dent.med.uni-muenchen.de

\section{Literatur}

[1] Welbury R, Raadal M, Lygidakis NA. EAPD guidelines for the use of pit and fissure sealants. Eur J Paediatr Dent 2004; 5: 179-184

[2] Toumba K], Twetman S, Splieth C et al. Guidelines on the use of fluoride for caries prevention in children: an updated EAPD policy document. Eur Arch Paediatr Dent 2019; 20: 507-516

[3] Ahovuo-Saloranta A, Forss $\mathrm{H}$, Walsh $\mathrm{T}$ et al. Sealants for preventing dental decay in the permanent teeth. Cochrane Database Syst Rev 2013; (3): CD001830

[4] Kühnisch J, Mansmann U, Heinrich-Weltzien R et al. Longevity of materials for pit and fissure sealing-results from a metaanalysis. Dent Mater 2012: 28: 298-303

[5] Kühnisch J, Bedir A, Lo Y-F et al. Meta-analysis of the longevity of commonly used pit and fissure sealant materials. Dent Mater 2020; 36: e158-e168

[6] Beauchamp J, Caufield PW, Crall J] et al. Evidence-based clinical recommendations for the use of pit and fissure sealants: a report of the American Dental Association Council on Scientific Affairs. J Am Dent Assoc 2008; 139: 257-268

[7] Irish Oral Health Services Guideline Initiative. Pit and fissure sealants: evidence-based guidance on the use of sealants for the prevention and management of pit and fissure caries. 2010. Im Internet (Stand: 23.02.2021): https://www.ucc.ie/ en/ohsrc/publications-guidelines/

[8] Smallridge J. UK National Clinical Guidelines in Paediatric Dentistry. Use of fissure sealants including management of the stained fissure in first permanent molars. Int J Paediatr Dent 2010. doi:10.1111/j.1365-263X.2009.01035.X

[9] NSW Ministry of Health. Policy Directive - Pit and fissure sealants: Use of in Oral Health Services NSW. North Sydney (Australia) 2013: PD2001325

[10] S3-Leitlinie zur Fissuren- und Grübchenversiegelung, Langversion 2016, AWMF-Registernummer: 083/002. Im Internet (Stand: 23.02.2021): https://www.awmf.org/leitlinien/detail/ II/083-002.html

Bibliografie

Zahnmedizin up2date 2021; 15: 9-12

DOI 10.1055/a-1323-0637

ISSN 1865-0457

(c) 2021. Thieme. All rights reserved.

Georg Thieme Verlag KG, Rüdigerstraße 14,

70469 Stuttgart, Germany 